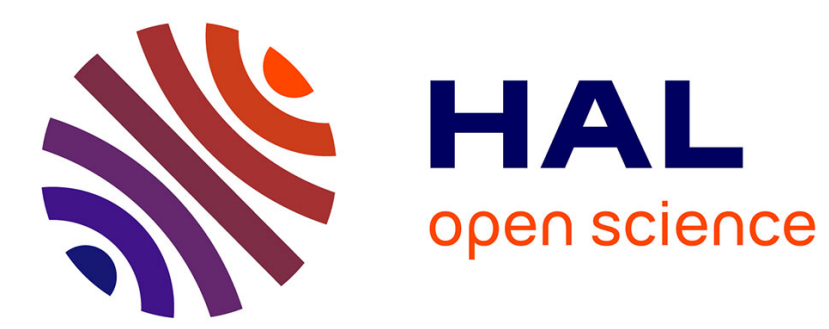

\title{
Temps verbal, aspect et point de vue: de la langue au discours
}

Jacques Bres

\section{To cite this version:}

Jacques Bres. Temps verbal, aspect et point de vue: de la langue au discours . Les cahiers de praxématique, 2003, 41, pp.55-84. hal-01340814

\section{HAL Id: hal-01340814 \\ https://hal.science/hal-01340814}

Submitted on 1 Jul 2016

HAL is a multi-disciplinary open access archive for the deposit and dissemination of scientific research documents, whether they are published or not. The documents may come from teaching and research institutions in France or abroad, or from public or private research centers.
L'archive ouverte pluridisciplinaire HAL, est destinée au dépôt et à la diffusion de documents scientifiques de niveau recherche, publiés ou non, émanant des établissements d'enseignement et de recherche français ou étrangers, des laboratoires publics ou privés. 
Jacques Bres

Praxiling, FRE CNRS 2425

Discours, textualité et production de sens

Montpellier III

jacques.bres@univ-montp3.fr

article paru dans Cahiers de praxématique 41, 2003, 55-84

\section{résumés}

La notion de point de vue est, dans un ensemble de travaux récents, fortement sollicitée pour l'analyse des temps verbaux. Après avoir montré que le vocabulaire de la perception fonctionnait comme une métaphore permettant d'appréhender la catégorie abstraite de l'aspect, on propose une analyse critique de l'usage de la notion narratologique de point de vue dans la littérature sur la question. On explicite ensuite les liens entre l'instruction aspectuelle délivrée par le temps verbal en langue et la construction d'un point de vue qui peut lui être associé en discours. L'étude est conduite sur l'imparfait et le passé simple.

The notion of viewpoint is used to a large extent in a set of recent works for the analysis of verbal tenses. We first show that perception lexicon works like a metaphor enabling one to grasp the abstract category of aspect. Next, we propose a critical analysis of the narratological notion of viewpoint which is appealed to in the literature on this topic. Lastly we explain the relations between the aspectual instruction given by the referential function of one tense and the construction of a viewpoint which may be associated to its pragmatic function. The study is based on two french tenses : the imparfait and the passé simple.

\section{Temps verbal, aspect et point de vue : de la langue au discours}

Le point de vue est, dans un ensemble de travaux récents (Fleischman 1991, Vogeleer 1994, Sthioul 1998, Labelle 2002, Rabatel 2003), fortement sollicité dans l'analyse des temps verbaux. Cette notion serait-elle en passe de devenir un nouveau paradigme explicatif de la temporalité ? Pour répondre à cette question, après avoir fourni deux précisions concernant l'acception de la notion de point de vue, et son association à la dimension aspectuelle du temps verbal (point 1.), je tâcherai de pointer la pertinence comme les limites de la métaphore scopique (point 2.). J'expliciterai ensuite les rapports entre aspect et point de vue tels qu'ils sont décrits par les recherches sur cette question (point 3.), avant de développer la façon dont j'articule ces deux notions à partir du concept d'actualisation, dans les cadres théoriques de la linguistique praxématique (point 4.).

\section{Précisions}

\subsection{Point de vue : narratologie, polyphonie}

La notion de point de vue, on le sait, procède de la narratologie, où, synonyme de focalisation, elle a pour but de répondre à la question qui voit?. A la suite de Ducrot (1984), certains linguistes - notamment Nølke (1993), Rabatel (1998), ou Haillet (2002) - étendront la problématique du point de vue à celle de la polyphonie, associant, voire amalgamant, les questions qui voit? et qui parle?. Dans cet article, je me placerai dans l'optique de Genette 1972 qui distingue les deux questions, et traiterai donc du problème du point de vue 
essentiellement dans l'acception narratologique de la notion ${ }^{1}$. J'ajoute que je limiterai mon analyse aux deux temps de l'imparfait (Imp) et du passé simple (PS), avec quelques incursions du côté du simple past anglais : ce sont principalement - mais non exclusivement eux qui se sont vus approchés par l'explication en termes de focalisation.

\subsection{Point de vue et temps verbal : l'aspect et/ou le temps?}

La métaphore scopique affecte la dimension aspectuelle, mais non la dimension temporelle des temps verbaux. Remarquons qu'on pourrait pourtant concevoir les époques future et passée comme vues à partir du nunc du présent. C'est d'ailleurs ainsi que procède Weinrich lorsqu'il met en place, dans son système d'analyse des temps verbaux, la notion de perspective de locution. Ainsi dans le groupe des temps commentatifs, le présent implique une perspective de locution neutre ; le passé composé, une perspective rétrospective ; le futur, une perspective prospective (1964/1973: 69). Mais il s'agit là d'une exception. A ma connaissance, personne n'a suivi Weinrich sur ce chemin consistant à associer la dimension temporelle à la problématique du point de vue. C'est la dimension aspectuelle - et seulement elle - qui est concernée dans les travaux mentionnés initialement.

\section{De l'usage de la métaphore du point de vue pour décrire l'aspect verbal}

\subsection{Linguistique et narratologie : échange de bons procédés}

Remarquons qu'il fut un temps pas si lointain, celui où la linguistique était considérée comme la science pilote à la fin des années soixante, où la toute nouvelle science textuelle qu'était la narratologie empruntait sa terminologie à la linguistique, notamment au vocabulaire concernant le verbe. Pour ne prendre que deux travaux prototypiques de ces années-là :

- Genette (1972) définit et nomme certaines catégories du récit à partir de concepts de la linguistique de la phrase, et singulièrement du verbe : ainsi la notion de mode lui sert à traiter du qui voit? ; celle de voix, du qui raconte?.

- Todorov, lorsqu'il veut analyser les différents types de perceptions à travers lesquels sont vus les événements du récit parle... d'aspects (1968:141).

Juste retour des choses si j'ose dire, quelque vingt ans plus tard, c'est l'inverse qui se produit : ce n'est plus l'aspect ou le mode verbal qui servent à catégoriser la focalisation narrative, mais la focalisation qui est sollicitée dans la description du temps verbal... Comment expliquer cette inversion de la métaphore ? On pourrait penser à deux raisons :

- l'échec relatif de l'approche du temps verbal selon le paradigme aspectuo-temporel pousserait à chercher ailleurs des explications, notamment du côté de la textualité. L'analyse de la temporalité verbale en termes de point de vue viendrait rejoindre les explications textuelles en termes de plans (Weinrich 1964/1973) ou d'anaphore (Kamp et Rohrer 1983, Molendijk 1990, Berthonneau et Kleiber 1993 entre autres références).

- plus largement, la linguistique de la langue serait actuellement concurrencée par la linguistique textuelle ou la pragmatique, qui inciteraient à compléter, voire à remplacer, l'approche linguistique des temps verbaux par des approches discursives.

Ces explications ont certainement une part de vérité. Elles me semblent cependant devoir être complétées par la lecture des textes des linguistes traitant du temps verbal, dans lesquels on

\footnotetext{
${ }^{1}$ Non que je considère la question qui parle ? et, plus largement, celle de l'hétérogénéité énonciative, comme non pertinente pour l'étude des temps verbaux : j'analyse moi-même certains imparfaits, notamment le tour hypocoristique (Bres 2003), comme dialogiques. Je réserve pour un travail ultérieur l'analyse des relations du temps verbal avec la question du point de vue dans son acception polyphonique ; comme celle de l'articulation entre polyscopie et polyphonie.
} 
pourra saisir en acte la naissance de la métaphore scopique. Un tel travail génétique demanderait une recherche très documentée pour savoir à quel moment et en quels termes apparaît le champ lexical de la perception. Ce ne sera pas l'optique choisie : je tenterai d'appréhender principalement dans deux textes théoriques - Guillaume 1929, Comrie 1976 la pente qui conduit naturellement de la problématique de l'aspect à la métaphore du point de vue, via un certain nombre de termes descriptifs de cette dimension : représentation, vision, image.

\subsection{Glissements progressifs de l'aspect vers le point de vue}

L'aspect semble naturellement convoquer pour sa description le champ lexical de l'espace, et plus précisément, celui, connexe et hyponymique, de la perception.

Guillaume, lorsqu'il présente sa théorie de l'aspect simple à partir de marcher, figure par un segment le temps intérieur au procès, et parle de «représentation entièrement tensive » pour l'infinitif, de «représentation à la fois tensive et détensive » pour le participe présent, de «représentation entièrement détensive » pour le participe passé (1929: 16-17) (les italiques sont miennes). On aurait tort de penser que le terme de représentation décrit seulement les schémas proposés pour chaque forme. Représentation a un statut cognitif (pleinement, et avant l'heure), ce que confirme le terme d'image qui lui est associé : «l'aspect tensif [...] éveille dans l'esprit l'image même du verbe dans son déroulement. Mettre son chapeau par exemple montre le chapeau qui, conduit par la main, va à la tête » (op. cit.: 21). Par l'intermédiaire du verbe voir («si je dis : marchant, je vois l'action non pas seulement en devenir comme avec l'infinitif [...]»(op. cit.: 17) (le soulignement est de mon fait), représentation cède la place à vision :

la forme participiale [en -ant] (...) représente la tension accompagnée de détension, soit (...) une vision sensiblement plus réelle que la vision d'infinitif (op. cit. : 19)

Et quelques pages plus loin, Guillaume avancera même la notion de «point d'observation » (p. 21-22).

On pourrait mettre la présence du champ lexical de la perception au compte de la conception psychomécanique spatialisante du temps verbal. Ce serait très insuffisant: aspect et visualisation ont partie liée, autrement que dans tel cadre théorique, comme le montre p. ex la façon dont Comrie et Langacker définissent l'aspect.

Comrie pose que « aspects are different ways of viewing the internal temporal constituency of a situation »; et indique, en note, que cette définition est fondée sur celle de Holt (1943 : 6) : « les manières diverses de concevoir l'écoulement du procès lui-même ». Il est intéressant de noter que Comrie a traduit concevoir par view. On note semblable passage du concevoir au voir chez Langacker qui définit ainsi le concept de «conceived time »: « time as an object of conceptualization, as it is viewed and portrayed $»(1987: 487)$.

La récurrence du champ lexical de la perception dans différentes théories fait sens. Il n'est sans doute pas innocent qu'aspect soit étymologiquement issu du latin aspicere, 'regarder'. A la différence de la dimension temporelle qui concerne le temps externe, l'aspect concerne le temps interne, qu'il représente de différentes manières. Ces différences de représentation sont conçues comme autant de façons de montrer ledit temps interne. La métaphore de la perception, loin d'être une approximation descriptive pré-scientifique, est à concevoir, en accord avec la grammaire cognitive et tout spécialement la théorie de la métaphore développée dans Lakoff et Johnson (1985), comme heuristique et pleinement légitime 
scientifiquement. Cette métaphore, usant des relations topologiques élémentaires, permet de structurer le domaine éminemment abstrait de l'aspect ${ }^{2}$.

Un pas de plus est franchi dans la mise en relation de l'aspect et du point de vue lorsque, dans le cas du couple PS / Imp, on glose ou remplace l'opposition aspectuelle global / sécant qui sert souvent à différencier ces deux temps, par la distinction vision externe / vision interne :

[l’Imp] permet de voir le procès de l'intérieur et non dans son unité (Martin 1971 : 96).

the perfective looks at the situation from outside, [...] whereas the imperfective looks at the situation from inside (Comrie $1976: 4$ ).

L'image est parfaitement juste: le PS (ou le perfectif) représente le temps interne de façon globale, ce qui ne peut se faire que si le point de saisie est extérieur au procès ; l'Imp (ou l'imperfectif) représente le temps interne dans son cours, en exclusion des bornes initiale et terminale, ce qui ne peut se faire que si le point de saisie est intérieur au procès. Les problématiques de l'aspect et du point de vue semblent alors se superposer : les signifiants aidant, on peut mettre en relation (i) vision interne de l'Imp et focalisation interne, et (ii) vision externe du PS et focalisation externe. Ce qui semble confirmé par le fait que l'Imp et la focalisation interne demandent à être associés, en récit, à la subjectivité d'un personnage, tout comme le PS et la focalisation externe (ainsi que la focalisation zéro) demandent à être associés à l'objectivité. Preuves complémentaires : Bally a fortement attiré l'attention sur la valeur subjective de l'imparfait (1912 : 601, cité in Rabatel 1998 : 42). Quant au PS, l'analyse proposée par Benveniste, qui définit ce temps, prototypique de l'énonciation historique, comme «temps de l'événement hors de la personne d'un narrateur», selon lequel «les événements semblent se raconter d'eux-mêmes » (1959/1966: 241), va dans le sens de la mise en relation PS / objectivité, hors de toute possible subjectivisation ${ }^{3}$.

Tout semble donc porter à associer aspect et point de vue, à identifier les problématiques, voire à remplacer la première par la seconde. Les problèmes ne tardent cependant pas à apparaître. L'association Imp- point de vue interne-subjectivité d'une part, et PS-point de vue externe-objectivité d'autre part, bute sur deux difficultés :

- Si l'Imp représente bien toujours le procès de l'intérieur, il peut le faire certes à partir du point de vue subjectif interne d'un personnage comme en (1), mais également en focalisation zéro à partir du point de vue omniscient et objectif du narrateur comme en (2) :

(1) Jean les regarda se perdre dans la nuit. La neige tombait plus épaisse, leurs vêtements confondus se liséraient d'un fin duvet blanc (Zola, La Terre).

(2) Maître Corbeau, sur un arbre perché, / tenait dans son bec un fromage./

Maître renard, par l’odeur alléché, / lui tint à peu près ce langage (La Fontaine, Fables)

Si tombait et se liséraient en (1) trouvent leur origine dans la subjectivité scopique de l'actant Jean, il n'en va pas de même de tenait en (2), que l'on ne peut que difficilement rapporter au point de vue du renard. Du coup, la notion narratologique de point de vue interne qui implique la subjectivité d'un personnage n'apparaît pas toujours appropriée pour définir l'Imp.

- Si le PS représente bien toujours le procès de l'extérieur, il peut le faire certes objectivement ou à partir du point de vue externe du narrateur omniscient comme en (3), mais

\footnotetext{
${ }^{2}$ Sur la pertinence de la métaphore de la perception pour décrire le temps verbal, on lira avec profit Gosselin (1996: 73-85).

${ }^{3}$ Position qu'adopte Fleischman lorsqu'elle avance que, à la différence de tous les autres temps verbaux qui impliquent un «experiencing-self », le passé simple rapporte un événement « as a completed past event and does so objectively », dans la mesure où il est «non-experiential, entirely detached from its speaker » $(1991: 31)$.
} 
également à partir du point de vue subjectif interne d'un personnage (cf. entre autres, Rabatel 1998 et 2003, Sthioul 1998, Leeman 2003, Nølke et Olsen 2003), comme dans (4) :

\author{
(3) Il voyagea. \\ Il connut la mélancolie des paquebots, les froids réveils sous la tente, l'étourdissement des \\ paysages et des ruines, l'amertume des sympathies interrompues. \\ Il revint (Flaubert : L'Education sentimentale).
}

(4) La yole semblait glisser. Des arbres se montrèrent sur l'île, dont la berge était si basse que les yeux plongeaient dans l'épaisseur des fourrés. On s'arrêta ; le bateau fut attaché (Maupassant, Une partie de campagne)

Les PS de (3) ne sauraient être vus à travers de filtre d'une quelconque subjectivité scopique. Il n'en va pas de même pour ceux de (4). L'incidence au temps, du fait du PS, du procès se montrer, est à mettre obligatoirement en relation avec le moment où le couple qui se déplace en barque perçoit lesdits arbres (qui, objectivement, ne sauraient se montrer : ils sont sur l'île, avant et après la perception qu'en a le couple); et il en va certainement de même pour $s$ 'arrêta (du fait de l'actantialisation par on. Le pronom ils effacerait la subjectivisation : «ils s'arrêtèrent »), et pour fut attaché (du fait du passif, l'actif aurait effacé la focalisation : «Henri attacha le bateau ») ${ }^{4}$. Ces PS procèdent du point de vue scopique des personnages. Du coup, la notion narratologique de point de vue externe qui implique la non-subjectivité d'un personnage n'apparaît pas toujours appropriée pour définir le PS.

On le voit donc, si la métaphore scopique semble globalement pertinente pour décrire l'aspect, dans le détail des faits la notion de point de vue dans son acception narratologique, ainsi que les catégories qu'elle développe (focalisations zéro, interne, externe) ne sont que partiellement adéquates, comme s'il y avait un décalage entre aspect et point de vue.

La mise en rapport des deux notions que j'ai esquissée à un niveau général, je vais maintenant voir comment elle se réalise dans différentes approches (point 3). Je développerai ensuite l'articulation que je propose (point 4).

\title{
3. Le rapport entre aspect et point de vue dans quelques théorisations
}

L'Imp et le PS sont-ils définissables par le point de vue, comme p. ex. Weinrich a tenté de faire la démonstration que ces temps l'étaient par la notion de mise en relief ? On va le voir, la tentation a été grande pour certains de procéder ainsi, mais elle a été repoussée : le point de vue, pour l'essentiel, apparaît non en substitution de l'explication en termes d'aspect, mais parallèlement à celle-ci, selon différentes modalités que nous allons analyser.

\subsection{Le point de vue en remplacement, ou en complément de la définition aspectuelle}

C'est sans doute chez Rabatel 1998 que la tentation de faire du point de vue une explication en dernière instance du fonctionnement du temps verbal - très précisément de l'Imp - est la plus forte. Suivons rapidement la convocation que l'auteur fait de cette hypothèse, comme sa révocation. Partant du point de vue, il se pose la question du rôle que joue l'Imp dans sa construction. Après avoir rappelé que Bally (1912) parle d'Imp subjectifs, il en infère que «l'imparfait est bien une forme verbale propre à traduire la subjectivité des perceptions » ( $1998: 45)$, avant de « se poser la question de savoir si (...) cette propension de l'Imp à exprimer le PDV ne serait pas la valeur fondamentale propre à ce temps » (op. cit. :

\footnotetext{
${ }^{4}$ Notons que l'actantialité est rapportée à des êtres non animés (c'est le mur qui se dresse, ce sont les arbres qui se montrent), à la forme pronominale pré-personnelle on; ou bien est effacée (le passif sans explicitation de l'agent), ce qui produit un effet de sens de conscience préréflexive.
} 
47). Et de citer différents emplois de l'Imp - de rupture, de dramatisation, d'événement, d'habitude... - passibles d'une analyse en termes de focalisation. L'envie est grande, on le sent, pour le spécialiste du point de vue, de faire le saut, mais Rabatel se l'interdit cependant, non sans quelque regret : «cette hypothèse, pour séduisante qu'elle soit, ne résiste pas à l'analyse. En réalité, ces valeurs ne tiennent pas au morphème d'imparfait lui-même, mais à l'opposition avec le PS ou le PC» (op. cit: 49). Poussant plus avant, dans une sorte d'autodialogisme avec ses propres hypothèses, il nie que l'Imp soit systématiquement le lieu d'un point de vue : «tout Imp n'exprime pas nécessairement un PDV » (ibid.). Et dans ses analyses, Rabatel associe le point de vue au second plan (cf. infra 4.2.), l'imparfait n'étant qu'un ingrédient possible de sa construction.

La tentative de faire du point de vue un élément définitoire du temps verbal se retrouve chez Labelle 2002, mais cette fois-ci, loin d'être (auto)interceptée, elle se voit conduite jusqu'à son terme: le point de vue apparaît comme un élément définitoire du temps verbal, complémentaire de la dimension aspectuelle.

Labelle reprend le cadre analytique proposé par Smith (1991, cf. infra 3.2.), pour le retravailler à propos du simple past anglais, défini par cet auteur comme perfectif au même titre que le PS, alors que le past progressive est défini comme imperfectif au même titre que l'Imp. Ce qui ne va pas, au dire de Labelle, sans poser un certain nombre de problèmes, dont le suivant. Partant des occurrences :

(5) Nero fiddled while Rome burned / was burning

(6) Néron joua / a joué / jouait du violon pendant que Rome *brûla / ?? a brûlé / brûlait

elle commente :

Comment se fait-il que le simple past perfectif puisse fonctionner avec while alors qu'en français, le passé simple et le passé composé sont exclus ? Comment le simple past peut-il assumer une partie du rôle d'un imperfectif? ( 2002 : 79)

Selon elle, l'analyse aspectuelle ne permet pas de répondre ; et la solution à cette difficulté passe par l'adjonction à la définition aspectuelle du temps verbal d'un élément relevant du point de vue : le simple past, qui peut présenter comme tout perfectif un procès d'un point de vue externe, peut de plus le présenter d'un point de vue interne comme dans (5), ce que ne peuvent faire les «perfectifs » français que sont le PS et le passé composé (6). Selon cette analyse, le point de vue n'interviendrait pas à un niveau linguistique différent de celui de l'aspect, mais serait un composant autonome, définitoire de la valeur du temps verbal : «le point de vue n'est pas surimposé : il fait partie de l'équation dans laquelle l'aspect est une variable distincte, dont C. Smith n'a pas tenu compte $»$ (op. cit. : 80). Ainsi le simple past serait à définir comme [points de vue externe et interne + perfectif], à la différence du PS qui serait de formule [point de vue externe + perfectif].

La solution est intéressante. Est-elle probante? Il me semble que l'on peut procéder différemment: l'asymétrie entre [while + simple past] et [pendant que $+* \mathrm{PS}]$ ne fait problème que pour autant que l'on se place dans un cadre théorique - celui de C. Smith - qui analyse le simple past et le PS comme relevant du même «viewpoint aspect », à savoir perfectif. C'est peut-être là que le bât blesse : le français, comme toutes les langues romanes, est une langue à imparfait, ce que n'est pas l'anglais, qui, en tant que langue germanique, est une langue à prétérit. Ce n'est que par approximation (de traduction) que l'on met en relation 
simple past et PS d'une part, et progressive past et imparfait d'autre part ${ }^{5}$. Linguistiquement, les systèmes sont radicalement différents. La solution à la question est à chercher du côté du système linguistique et des représentations aspectuelles qu'il construit : c'est certainement parce que l'anglais ne dispose que d'un prétérit (simple ou progressif) qu'il est compatible avec while ; c'est certainement parce que le français dispose d'un imparfait à côté du passé simple, que pendant que est incompatible avec le passé simple ${ }^{6}$. Ce que semble vérifier le fait qu'il en va de même dans des langues romanes comme le castillan, le catalan ou l'occitan, disposant de l'opposition Imp / PS. L'opposition perfectivité / imperfectivité, si elle permet de décrire adéquatement la distinction simple past / progressive past, ne permet pas de le faire pour le couple PS - Imp. L'instruction [perfectivité] doit être affinée, pour le PS, en [+ incidence]; l'instruction [imperfectivité] doit être affinée, pour l'IMP, en [- incidence] (cf. infra 4). Et c'est parce que le simple past est perfectif qu'il peut se combiner avec while; c'est parce que le PS est incident qu'il ne peut que difficilement se combiner avec pendant que. Point n'est donc besoin de rajouter un module point de vue pour rendre compte des emplois discursifs des temps verbaux, pourvu que la théorie aspectuelle à partir de laquelle on travaille ait suffisamment de finesse pour rendre compte des différents fonctionnements.

Après analyse, il semble donc que, qu'il s'agisse de l'Imp français ou du simple past anglais, la catégorie du point de vue (i) en confirmation du propos de Rabatel, ne saurait se substituer à celle de l'aspect; (ii) en contradiction avec le propos de Labelle, ne saurait jouer comme un module autonome, de même niveau que l'aspect et le complétant.

\subsection{Où le point de vue s'intrique avec l'aspect}

La catégorie du point de vue peut être présentée dans un rapport non de substitution ou de complémentation nécessaire, mais de forte intrication avec celle de l'aspect, comme dans Smith 1991. Cet auteur pose que l'aspect d'une phrase résulte de l'interaction de deux modules, à savoir le type de situation et le point de vue : "The aspectual meaning of a sentence results from interaction between two independent aspectual components, situation type and viewpoint » (intro, XVI). Le premier est marqué par le verbe, les arguments et les adverbes : il correspond à ce que certains nomment type de procès; le second, le viewpoint, est marqué morphologiquement par des affixes ou des formes spéciales : il correspond à ce qui est traditionnellement nommé aspect. La métaphore scopique est ici littéralisée : le terme d'aspect est remplacé par celui de viewpoint, et réservé hyperonymiquement pour désigner l'ensemble «situation type » (aspect lexical) + «viewpoint» (aspect grammatical). Elle est également filée :

Aspectual viewpoints function like the lens of a camera, making objects visible to the receiver. Situations are the objects on which viewpoint lenses are trained. And just as the camera lens is necessary to make the object available for a picture, so viewpoints are necessary to make the object

\footnotetext{
${ }^{5}$ Ce qu'un simple relevé des imparfaits d'une nouvelle de Maupassant mis en relation avec leur traduction anglaise infirme : comme l'a montré A. Patard dans un mémoire de DEA (« L'imparfait en contexte : l'un et le multiple »), l'imparfait français est traduit plus fréquemment par le simple past que par le progressive past.

6 Cette assertion est d'ailleurs à nuancer. Contrairement à ce qu'avance Labelle (mais également Leeman 2003 : 27), le PS peut également se conjoindre avec pendant que pourvu qu'il soit possible de considérer les deux procès mis en relation comme simultanés :
}

(7) Pendant que Chesnel s'habilla, Mme de Maufrigneuse dégusta la tasse de café à la crème que Brigitte lui servit (Balzac, Le Cabinet des Antiques).

On interprète alors non que s'habilla inclut dégusta, soit [s’habilla $\supset$ dégusta], mais que les deux procès sont simultanés, à savoir [s'habilla = dégusta]. 
available for a picture, so viewpoints are necessary to make visible the situation talked about in sentence (1991: 91)

Cependant si dans le métalangage viewpoint remplace aspect, dans les faits Smith met sous viewpoint ce qui est traditionnellement mis sous aspect. En effet, distinguant trois viewpoints, elle les définit et les distingue à partir de l'opposition aspectuelle classique perfective / imperfective (complétée de la catégorie de neutral), et non p. ex. à partir de l'opposition possible external viewpoint / internal viewpoint :

Perfective viewpoints focus on the situation as a whole, with initial and final points.

Imperfective viewpoints focus on part of a situation, including neither initial nor final point.

Neutral viewpoints are flexible, including the initial point of a situation and at least one internal stage (where applicable) (op. cit. : 6).

Remarquons cependant que les notions de perfective et d'imperfective s'appliquent non au procès lui-même mais à la «situation » ${ }^{7}$, c'est-à-dire non plus à une unité de langue (le procès) mais à une unité d'actualisation de la langue en discours, construite par le procès et ses actants ${ }^{8}$. La perspective est clairement pragmatique : «viewpoint gives the receiver a full or partial view of a situation» (op. cit.: 5); alors que dans une approche strictement aspectuelle, on dira que ce que l'aspect donne à voir de telle ou telle façon, ce n'est pas directement la situation mais le temps interne impliqué par le verbe. Et c'est selon que le temps interne est saisi par l'aspect globalement ou dans son cours que la situation à laquelle renvoie le procès sera vue globalement ou partiellement.

Les propositions d'analyse de Smith littéralisent la métaphore scopique au niveau du métalangage descriptif (viewpoint, view, focus), sans pour autant renoncer au vocabulaire spécifique de l'aspect (perfective, imperfective). Satisfaisantes dans une perspective pragmatique qui se préoccupe de savoir à quoi sert tel morphème dans la communication d'un énoncé - effectivement l'aspect verbal sert indirectement à montrer la scène dans sa globalité, dans son cours, ou au-delà de son terme ${ }^{9}-$ ces propositions le sont moins pour qui se préoccupe de tenter de comprendre par quelles interactions précises entre morphèmes on passe de la langue au discours. Je reviendrai sur ce point lorsque je présenterai ma propre hypothèse.

\subsection{Alternance des temps et alternance du point de vue}

Dans une perspective également pragmatique, on peut décrire non le temps verbal, via l'aspect, comme servant à présenter la scène sous un certain point de vue, mais l'alternance des temps verbaux comme servant pragmatiquement à signifier l'alternance du point de vue. Fleischman 1991 développe ce type d'analyse. Distinguant deux types de fonctions: « referential functions (e. g. past or future time reference, perfective or imperfective aspect), which I take to be ontogenetically primary, and pragmatic functions, which develop as secondary extensions of the basic grammatical meanings » (op. cit. : 47), elle fait l'hypothèse que l'alternance des temps a pour fonction pragmatique de distinguer différentes focalisations à travers lesquelles le monde raconté est présenté (op. cit. : 45). Il en irait ainsi de l'alternance PS / Imp dans l'occurrence suivante de L'Expiation (Hugo, Les Châtiments) qui intéresse les linguistes au moins depuis Yvon (1926) :

\footnotetext{
${ }^{7}$ C'était également le cas chez Comrie.

${ }^{8}$ Unité correspondant au petit drame de Tesnière, au spectacle en praxématique, à la scène chez Victorri et Fuchs 1996.

${ }^{9}$ Aspect accompli que ne prend pas en compte Smith.
} 
(8) Au cours de la bataille de Waterloo, Napoléon distingue dans le lointain une silhouette qu'il croit être celle de son général Grouchy, mais erreur il s'agit de celle du prussien Blücher.

Soudain, joyeux, il dit : Grouchy ! - C'était Blücher.

L'espoir changea de camp, le combat changea d'âme.

(Hugo, L'Expiation in Les Châtiments)

L'imparfait était exprimerait le point de vue du narrateur omniscient qui vient corriger l'erreur de perception du personnage (dit). L'alternance PS / Imp exprimerait un changement de point de vue (du personnage au narrateur).

L'exemple me paraît mal choisi : il me semble que ce n'est pas parce qu'est imputé à Napoléon, en discours direct, l'énoncé « Grouchy », que pour autant le verbe introducteur au PS dit adopte le point de vue de ce personnage. Loin d'être filtré par la conscience du personnage, ce PS procède du seul narrateur, tout comme l'Imp était. L'alternance temporelle ne recouvre ici aucun changement de point de vue (cf. infra 4.).

Les autres exemples d'alternance PS / Imp qu'analyse S. Fleischman concernent la transition discours du narrateur > discours indirect libre du personnage, à savoir le point de vue dans l'acception polyphonique, comme dans :

(9) Ensuite Arnoux parla d'une cuisson importante que l'on devait finir aujourd'hui, à sa fabrique. Il voulait la voir. Le train partait dans une heure (Flaubert, L'Education sentimentale)

Ici effectivement le changement de temps (parla > devait, voulait, partait) correspond à un changement de point de vue, «from the external perspective of the narrator to the inner speech and thoughts of the focalizing characters » (op. cit. : 35). Pour autant peut-on dire que c'est là sa fonction textuelle ? D'autre part, si l'on adopte sans la critiquer l'hypothèse de Fleischman, l'alternance PS / Imp marquerait en (8) le passage du point de vue du personnage à celui du narrateur; en (9), la transition du point de vue du narrateur à celui du personnage $^{10}:$ l'alternance des temps marquerait-elle indifféremment l'alternance du point de vue?

L'hypothèse de S. Fleischman selon laquelle la fonction textuelle de l'alternance PS / Imp serait de marquer l'alternance du point de vue apparaît comme bien fragile :

- dans la majorité des cas, l'alternance PS / Imp ou Imp /PS n'exprime pas de changement de perspective : c'est le cas de (8) comme de (2);

- il existe des alternances de point de vue (au sens polyphonique de qui parle ?) que n'accompagne pas l'alternance temporelle :

(10) Le soir, Gervaise avouait franchement en riant qu'elle en serait tombée malade si on l'avait empêchée d'avoir la boutique. Toutefois, avant de dire : C'est fait ! elle voulait emmener Coupeau voir les lieux et tâcher d'obtenir une diminution du loyer.

- Alors, demain, si ça te plaît, dit son mari (Zola, L'Assommoir).

Soient les deux imparfaits avouait et voulait : le premier, en emploi dit narratif, ne fait l'objet d'aucune focalisation; le second, par contre, du fait du cotexte, très précisément du discours direct du mari de Gervaise, qui se présente conversationnellement comme un tour réactif répondant à un tour initiatif, présuppose que la proposition « elle voulait emmener Coupeau (...)» est du discours indirect libre. Voulait est donc à rapporter au personnage de Gervaise. La succession avouait, voulait recouvre donc un changement de perspective (narrateur > personnage).

\footnotetext{
${ }^{10}$ Phénomène que Fleischman n'a pas relevé.
} 
De sorte que, pas plus que les temps verbaux ne sont liés à l'expression de tel ou tel point de vue, il me semble que l'alternance PS / Imp, quel que soit son ordre, n'a pas pour fonction d'exprimer le changement de point de vue.

\subsection{Point de vue et usages interprétatifs}

Si les temps verbaux ne sont pas définissables par le point de vue, s'ils ne sont pas associés de façon biunivoque à tel point de vue, si leur alternance ne marque pas forcément l'alternance du point de vue, alors quels liens peuvent être établis entre les deux notions ? B. Sthioul (Sthioul 1998, de Saussure et Sthioul 1999, Sthioul 2000), avançant que «tous les temps verbaux se prêtent, certes avec plus ou moins de facilité, à des effets de sens [de subjectivisation]»(2000: 80), propose, en appui sur la théorie de la pertinence, une articulation très intéressante temps verbal / point de vue selon laquelle le temps verbal est lié de façon forte au point de vue dans certains de ses usages : les usages interprétatifs. Rappelant la distinction faite par la théorie de la pertinence entre usages descriptifs, dans lesquels l'énoncé décrit un état de fait, et usages interprétatifs dans lesquels l'énoncé représente une pensée à propos d'un état de fait, Sthioul pose que :

- en usage descriptif, les temps verbaux ne sont pas associés à la production d'un effet de subjectivisation; la séquence est non-focalisée, «les énoncés rendent compte d'événements et de situations sans passer par le filtre d'un sujet de conscience distinct du locuteur au moment de l'énonciation » $(2000: 81)$;

- en usage interprétatif, les temps verbaux sont associés à la production d'un effet de subjectivisation; la séquence est focalisée, «le locuteur retransmet les pensées ou les sensations d'une instance distincte » (ibid.).

L'interprétation en termes d'usage interprétatif intervient en cas d'échec de l'interprétation en termes d'usage descriptif. Illustrons l'hypothèse sur l'Imp et sur le PS.

L'imparfait est défini par deux instructions : le procès est appréhendé de l'intérieur, à partir d' ' un point de perspective $\mathrm{P}$, abstrait et sous-déterminé, inclus dans le procès » (de Saussure et Sthioul 1999: 176) $(\mathrm{P} \subset \mathrm{E})$; et l'appréhension du procès est déconnectée du moment de l'énonciation $(\mathrm{P} \# \mathrm{~S})$. En usage descriptif, le destinataire attribue à $\mathrm{P}$ une valeur d'antériorité par rapport à $\mathrm{S}$, et d'inclusion temporelle. C'est prototypiquement le cas pour l'énoncé :

(11) Marie entra dans le bureau du président. Une copie du budget était sur la table (cité in Sthioul $2000: 86)$.

Le point de perspective est à rattacher au point référentiel introduit par entra, qui est inclus dans était.

Mais parfois, pour des raisons contextuelles, l'interprétation descriptive est bloquée. Le destinataire dote alors $\mathrm{P}$ d'une autre valeur : il l'assimile « à un moment de conscience $\mathrm{C}$, relativement auquel il peut obtenir de manière consistante l'inclusion dans le procès »(de Saussure et Sthioul 1999 :178). Sthioul cite plusieurs cas de blocage de l'usage descriptif qui nécessitent de recourir à l'usage interprétatif: imparfaits préludique, hypocoristique, hypothétique, narratif. Je ne présente que ce dernier, à partir de l'exemple classique :

(12) Pierre alluma la lampe. La lumière donnait à la pièce un air de tristesse désolée.

Dans ce cas, on ne peut interpréter donnait comme incluant le précédent point référentiel, à savoir alluma, puisque nos connaissances du monde posent la succession [allumer < donner un air]. Il faut donc construire un point de perception : dans le cas de (12), il s'agira du point de vue interne de Pierre regardant « la tristesse désolée » de la pièce, qui peut être inclus dans 
donnait. Sthioul appuie son hypothèse sur le fait que la bizarrerie de l'imparfait dans (13) (repris à Tasmowski-de Ryck 1985) :

(13) ?Paul tomba raide mort. Sa femme appelait à l'aide,

tiendrait au fait que la mort soudaine de Paul «l'empêche d'être un candidat adéquat à l'observation des événements ultérieurs » $(1998: 215)$.

L'analyse conduite sur le passé simple est similaire. Affectant au PS l'instruction de progression, Sthioul fait remarquer qu'une occurrence comme :

(14) Paul sortit. Dehors, il fit froid

est problématique dans la mesure où la sémantique du PS demande de construire une relation de succession [sortit < fit froid], alors que nos connaissances du monde demandent une relation d'inclusion ([sortir $\subset$ faire froid]), dans la mesure où il faisait froid avant et après que Paul ne sorte. L'acceptabilité de (14) est sauvée si l'on positionne le point de vue de Pierre : «l'énoncé pointerait sur le moment où le personnage prend conscience d'une situation à propos de laquelle, par ailleurs, on peut inférer en vertu de nos connaissances du monde qu'elle a débuté antérieurement $»(2000: 90)$.

L'hypothèse explicative proposée par Sthioul est puissante : elle s'applique à l'ensemble des temps verbaux, et s'appuie sur la distinction théorique des usages descriptifs / interprétatifs productive par ailleurs. Elle n'emporte cependant pas mon adhésion pour trois raisons :

- Pour ce qui est de l'imparfait : est-ce bien l'impossibilité de subjectivisation du fait de la mort de Pierre qui est comptable du caractère bizarre de (13) ? Je n'en suis pas sûr. Remplaçons la première phrase de cet exemple :

(15) ?Paul tomba et se cassa la jambe. Sa femme appelait à l'aide.

Paul est désormais bien vivant et sa conscience peut parfaitement médiatiser l'appel au secours de sa femme. Et pourtant (15) est tout aussi bizarre que (13) ${ }^{11}$. S'il suffisait d'un point de vue pour rendre acceptable un imparfait, alors (15) ne devrait pas poser problème. Ce qui n'est pas le cas. De sorte que l'explication de l'imparfait narratif en termes de point de vue, fût-ce en appui sur la théorie de la pertinence, ne semble pas pouvoir rendre vraiment compte du caractère problématique de l'imparfait dans (13).

- A l'inverse, de très nombreux imparfaits narratifs qui introduisent un nouveau point référentiel ne sauraient s'expliquer en termes de subjectivisation, alors même qu'ils sont parfaitement acceptables. Je cite entre autres occurrences, celle-ci :

(16) Ils se glissèrent à tâtons entre les lits occupés dans la grande salle crépie à la chaux, où fumait une veilleuse au fond d'une niche sur la muraille; et toute la nuit serrés l'un contre l'autre, ils étouffaient leurs baisers et leurs rires en entendant ronfler, geindre de fatigue ces compagnons, dont les bourgerons, les lourdes chaussures de travail traînaient tout près de la robe de soie et des fines bottes de la Parisienne.

Au petit jour, une chatière s'ouvrit au bas du large portail (...) (Daudet, Sapho).

Qui pourrait bien être à l'origine de la perspective à partir de laquelle serait vu l'acte qu'actualise l'imparfait étouffaient? Certainement pas les « camarades » du jeune couple qui partagent cette chambre commune : leurs ronflements attestent qu'ils ne sauraient ni voir ni surtout entendre les baisers des deux héros...

\footnotetext{
${ }^{11}$ Cette remarque critique était déjà formulée dans Bres 1999, ainsi que dans Berthonneau et Kleiber 1999 : 139, note 14 .
} 
- Enfin, et peut-être surtout : l'effet de subjectivisation peut très bien être produit en total accord avec les instructions de l'imparfait ou du passé simple, telles que les pose Sthioul. Reprenons (11) sous (17) :

(17) Marie entra dans le bureau du président. Une copie du budget était sur la table.

Si on est bien d'accord que le point de vue de Marie n'est pas obligatoire dans l'interprétation de cet énoncé comme le prouve (18) :

(18) Marie entra dans le bureau du président. Une copie du budget était sur la table, mais elle ne la vit pas.

il n'empêche que c'est majoritairement l'interprétation subjective qui est actualisée en (17), et que, dans ce cas comme dans celui où l'énoncé est non focalisé car procédant du seul locuteur, les instructions sont parfaitement satisfaites : était inclut le point référentiel précédant entra.

Et il en va de même pour le PS. L'énoncé, tout en étant en plein accord avec les instructions sémantiques du PS, peut demander de construire un point de vue à travers le filtre duquel les faits racontés semblent bien être vus. Reprenons (4) sous (19) :

(19) La yole semblait glisser. Des arbres se montrèrent sur l'île, dont la berge était si basse que les yeux plongeaient dans l'épaisseur des fourrés. On s'arrêta; le bateau fut attaché (Maupassant, Une partie de campagne).

Les PS se montrèrent, s'arrêta, fut attaché, alors même qu'ils sont en parfait accord avec l'instruction [+ progression] par laquelle Sthioul définit le PS - à savoir que l'on comprend [se montrèrent < s'arrêta < fut attaché] - semblent bien être vus à travers le regard du couple embarqué sur la yole.

Il semble donc que le cadre théorique proposé par Sthioul rencontre certaines difficultés. La nécessité de construire un point de vue scopique subjectif ne procède pas (forcément) d'une difficulté à construire du sens à partir des instructions données par le temps verbal, dans le cadre des « usages descriptifs».

Les analyses que nous avons présentées, qu'elles articulent aspect et point de vue en termes de complément (Labelle 2002), de parallélisme dans l'alternance (Fleischman 1991) ou de fonctionnement interprétatif (Sthioul 2000), ne semblent pas déterminantes pour rendre compte du rapport entre les deux notions ${ }^{12}$. Je vais expliciter maintenant la façon dont je conçois, dans les cadres théoriques de la linguistique de l'actualisation qu'est la praxématique, les liens et relations entre aspect et point de vue.

\section{De l'aspect côté cour, du point de vue côté jardin}

Malgré la métaphore cognitivement pertinente qui pousse à décrire l'aspect en termes scopiques, aspect et point de vue me semblent relever de deux ordres différents : de l'ordre de la langue pour l'aspect, de celui du discours pour le point de vue. Autrement dit, l'aspect entre en jeu précocement dans l'acte d'actualisation, le point de vue plus tardivement. Ce qui rend

\footnotetext{
${ }^{12}$ Je ne prends pas en compte ici l'article de Vogeleer 1994, malgré son titre - « Le point de vue et les valeurs des temps verbaux »- qui semble pourtant le placer en plein cœur de notre recherche. Et ce, pour la raison suivante : le point de vue, chez cet auteur, correspond non à la notion narratologique classique mais à un concept spécifique : la « relation point de vue » qui « se présente sous la forme d'une relation cognitive, de nature épistémique (il s'agit notamment de la relation savoir) ou perceptuelle (notamment la relation voir), qui relie un individu (...) à l'information décrite dans une phrase contextualisée » (1994 : 40). L'article est consacré à distinguer les valeurs épistémiques et perceptuelles des temps verbaux, notamment du PS et de l'Imp.
} 
compte de ce que ces deux temps, distincts aspectuellement, puissent être associés - certes de façon différente - aux mêmes effets de sens « objectifs » et «subjectifs ». Je vais tâcher de montrer concrètement comment interviennent, dans l'acte d'actualisation, l'aspect, puis le point de vue ; j'expliquerai ensuite pourquoi l'Imp a plus d'affinités avec cette catégorie que le PS.

\subsection{De l'aspect au point de vue dans l'acte d'actualisation}

J'analyserai deux occurrences : la première, précédemment citée, qui ne demande pas de construire un point de vue subjectif, la seconde qui le demande.

(a) Reprenons (8) sous (20) :

(20) Soudain, joyeux, il dit : Grouchy ! - C'était Blücher.

En langue, la valeur aspectuelle du PS est ([+ tension], [+ incidence]) ; celle de l'imparfait, ([+ tension], [- incidence]. Les deux temps verbaux se différencient donc par la catégorie de l'incidence. Cette notion, que j'ai empruntée à Gustave Guillaume pour la retravailler, concerne principalement le mode d'inscription du temps interne au procès sur l'imaginaire ligne du temps que construit le discours. Le temps verbal peut demander de se représenter le temps interne du procès :

- en incidence, c'est-à-dire s'inscrivant sur ladite ligne du temps sans la traverser, en seul accomplissement, depuis son terminus a quo jusqu'à son terminus ad quem. On dira qu'est délivrée l'instruction [+ incidence] : c'est le cas du PS ;

- comme s'inscrivant sur la ligne du temps en la traversant, en une incessante conversion de l'accomplissement en accompli, en un point situé au-delà de son terminus a quo et en-deçà de son terminus ad quem. On dira qu'est délivrée l'instruction [- incidence] : c'est le cas de l'Imp.

Dans l'actualisation de l'énoncé, l'aspect concerne donc le temps interne impliqué par le procès. Dans le cas de (20), le PS demande de se représenter le temps impliqué par le verbe dire qu'il actualise de façon incidente; et l'Imp, le temps impliqué par être de façon non incidente. Attribuer plus de pouvoir à l'aspect, c'est lui imputer des éléments de sens dans lesquels il est au mieux partie prenante.

A un deuxième niveau, d'actualisation plus avancée, celui des relations chronologiques interphrastiques, le PS, de par sa structure aspectuelle, a des affinités avec la relation de progression; et l'Imp avec la relation d'inclusion (Bres 2004b). Si le cotexte et nos connaissances du monde le permettent, ce sont ces relations qui seront actualisées. Dans le cas qui nous intéresse,

- le PS dit, en accord avec nos connaissances et avec le cotexte (notamment l'adverbe soudain), introduit un nouveau point référentiel ;

- l'imparfait était peut parfaitement inclure dans le temps qu'il implique le point référentiel de dit : avant, après et même au moment où Napoléon dit "Grouchy », il s'agit en fait de Blücher. Ce que confirment les types de procès : dire est un achèvement; être, un état : le premier peut sans problème être inclus dans le second. La question du point de vue ne se pose à vrai dire pas : sans difficulté pour la cohérence textuelle, le narrateur omniscient impute au personnage un dire («Grouchy ») qu'il montre tout de suite après comme erroné en rectifiant l'identification. L'erreur d'analyse de S. Fleischman (supra 3.3.) me semble tenir à ce qu'elle a confondu celui qui dit «Grouchy », à savoir Napoléon, et celui qui dit que Napoléon dit « Grouchy », à savoir le narrateur. Si effectivement un même locuteur ne peut dire, dans le même temps, que c'est Grouchy et que c'est Blücher, rien n'empêche par contre de rapporter à une seule et même instance, le narrateur, le fait de rapporter la parole d'un 
personnage (dit) pour en corriger aussitôt l'adéquation à la réalité (était), sans que l'ombre d'un point de vue subjectif ne se profile... Dans l'occurrence (20), aucun élément cotextuel ne la sollicitant, la construction d'une subjectivité scopique n'est pas entreprise.

(b) Alors que l'aspect est obligatoire - il est lié à la définition du temps verbal : quel que soit son emploi en discours le PS donne l'instruction [+ incidence], l'imparfait l'instruction [incidence] -, la subjectivité scopique est un phénomène discursif procédant de l'interaction de divers ingrédients, qui peut donc, selon le cotexte, être absent comme dans (18), présent ou absent comme en (17), obligatoirement présent comme en (19) ou en (21) :

(21) Justin faillit se jeter dans un mur qui se dressa devant lui après un tournant, évita de justesse un camion (Triolet, Luna-Park).

Le PS demande de concevoir le temps impliqué par le procès de type achèvement se dresser en seule incidence à partir de sa borne initiale. Cette représentation, qui résulte de l'interaction entre le procès se dresser et l'instruction du PS qui l'actualise, fait difficulté au niveau ultérieur de l'actualisation phrastique: de par nos connaissances du monde, nous savons qu'un mur ne peut se dresser instantanément, que ledit mur était à cet endroit avant que Justin ne passe et y demeurera après son passage... La discordance entre la représentation du verbe se dresser au PS et son contexte trouve solution dans l'effet de sens de subjectivisation : c'est Justin qui perçoit le mur dans son érection subite, ce que confirme le circonstant devant lui. Le surgissement (se dressa) est à mettre au compte du point de vue scopique du personnage.

Dans le temps opératif de production de l'énoncé, l'aspect intervient obligatoirement et précocement : il actualise la façon dont le temps interne du procès est à concevoir. Le point de vue se construit facultativement et plus tardivement : en interaction avec la représentation fournie par le verbe ainsi actualisé par l'aspect (et le temps), le co(n)texte (notamment le praxème verbal lui-même, l'actant sujet, les relations chronologiques interphrastiques) peut demander de construire une subjectivité scopique, autre que celle du locuteur (ou du narrateur), à partir de laquelle la scène est perçue.

\subsection{Les relations du PS et de l'Imp avec le point de vue : et plus si affinités...}

Est-ce à dire que les deux phénomènes de l'aspect et du point de vue soient totalement indépendants ? La réponse est négative : il est évident que l'Imp a plus d'affinités avec la subjectivité scopique que le PS. Qu'est-ce qui rend compte de ce fait?

Deux explications ont été fournies de cette prédisposition de l'imparfait pour la mise en place d'un point de vue subjectif, comme de cette résistance tendancielle du PS à cette association : la première, par Rabatel 1998 ; la seconde, par Nølke et Olsen 2003.

(a) Rabatel (1998: 30-41), reprenant une analyse développée par Combettes 1992, qui développait lui-même Weinrich 1964/1973, définit le premier plan comme celui du récit objectif de l'action; l'arrière-plan, comme celui où peuvent se développer les perceptions subjectives. Ce serait donc parce que l'imparfait est le temps de l'arrière-plan, qu'il serait aisément focalisable ; et ce serait parce que le PS est le temps du premier plan qu'il ne serait pas - ou plutôt peu - focalisable. La difficulté de l'explication réside dans ce peu : les occurrences de PS «subjectif » contredisent en effet l'analyse liant obligatoirement point de vue et second plan. Conscient de cette difficulté mais soucieux de conserver ce lien, Rabatel n'a d'autre solution que de faire valoir que le PS, temps le plus souvent du premier plan, est, dans les occurrences où il s'origine dans une subjectivité, " de second plan » (p.37).

Pour ingénieuse qu'elle soit, cette solution me semble faire problème pour deux raisons :

- aucune preuve linguistique n'est apportée à la proposition selon laquelle le PS serait « de second plan » lorsqu'il est focalisable. On ne peut guère s'empêcher de penser que c'est 
le choix d'un type d'analyse, celui des plans, bien plus que les faits linguistiques, qui l'exige : le PS « subjectif » n'est-il pas conçu comme de second plan parce que l'analyse a auparavant établi un lien d'exclusivité entre second plan et focalisation?

- l'analyse du PS subjectif en termes de second plan va à l'encontre de l'impression de lecture selon laquelle le PS subjectif appartient bien, comme le PS objectif, au premier plan. Relisons (19) : les procès se montrèrent, s'arrêta, fut attaché présentent les événements selon l'ordre progressif : [se montrèrent < s'arrêta < fut attaché], ils font progresser l'histoire : il semble plus que contre-intuitif de considérer qu'ils nous font passer du premier plan à l'arrière-plan.

(b) Nølke et Olsen 2003 font appel à une autre distinction - deixis vs anaphore - pour rendre compte de la facilité du mariage de l'Imp avec le point de vue, comme du peu d'appétence du PS pour ce type de lien. Partant de l'analyse selon laquelle le PS serait déictique et l'imparfait anaphorique, ils en infèrent que :

- le PS - du fait que le calcul déictique s'effectue à partir du locuteur - tend à représenter le point du vue de celui-ci. Pour qu'il en soit différemment, il faut qu'une autre conscience soit explicitement posée ;

- l'imparfait, à l'inverse, de par son anaphoricité, sollicite fortement (mais pas nécessairement) la médiation d'une conscience à laquelle rapporter le procès qu'il actualise.

Il me semble que c'est aller un peu vite en besogne que de conjoindre PS et deixis d'une part, Imp et anaphore de l'autre. Les spécialistes de la question sont très réservés à cet endroit (cf. notamment Kleiber 1993). Sans développer ce point très controversé, on peut dire que (i) l'Imp, comme le PS, a une dimension déictique : si, dans une perspective temporaliste, je considère l'imparfait comme un temps du passé, alors il situe l'événement par rapport au nunc, c'est-à-dire qu'il a, au moins en partie, un fonctionnement déictique ; (ii) de même que le PS, comme l'Imp mais certainement de façon différente, à savoir moins fortement, a une dimension anaphorique dans la mesure où il sollicite un intervalle de validation (Kleiber $2003: 10)$.

De sorte que l'explication de la différence d'affinité de l'Imp et du PS avec le point de vue, en termes d'opposition anaphore / deixis, pour avoir une réelle pertinence, devra être singulièrement affinée.

(c) Il apparait donc que les explications en termes de distinction des plans ou d'opposition anaphore / deixis ne sont pas concluantes. L'hypothèse que j'avance est fort simple : la différence d'affinité de l'Imp et du PS avec la focabilité tient à leur structure aspectuelle. Tous les linguistes ont noté que l'imparfait avait une autonomie textuelle bien moindre que le PS :

L’imparfait est un temps "dépendant". Si je dis : "hier il a plu", la phrase se suffit à elle-même. Si je dis: "hier, il pleuvait", mon interlocuteur sait qu'il faut suppléer quelque chose. Ma phrase peut signifier, d'après l'intonation : "il pleuvait (à cette même heure)", ou : "il pleuvait (et j'ai dû renoncer à ma promenade)", etc. (Brunot et Bruneau 1947/1969 : 331).

C'est même en se fondant sur cette dépendance que l'Imp est appréhendé comme un temps anaphorique. Mais à quoi tient cette différence d'autonomie ? Selon moi, à l'instruction aspectuelle que délivre chacune des deux formes. Le PS, en inscrivant sur la ligne du temps que construit le discours le temps interne représenté par le procès qu'il actualise à partir de sa borne initiale jusqu'à sa borne terminale - c'est-à-dire en incidence -, assure à cette inscription un fort enracinement, et par conséquent une grande indépendance : une phrase au PS est relativement autonome.

Au contraire l'imparfait, en inscrivant le temps interne représenté par le procès qu'il actualise au-delà de sa borne initiale et en deçà de sa borne terminale - c'est-à-dire de façon non incidente -, l'enracine faiblement, et par conséquent tend à l'assujettir à d'autres éléments du 
co(n)texte : l'imparfait a besoin de quelque chose d'autre. Ce supplément d'ancrage est fourni le plus souvent, en récit, par un procès au PS, avec lequel l'imparfait établit une relation d'inclusion, comme dans l'occurrence (20), dans laquelle était est interprété comme incluant dit $:[$ dit $\subset$ était $]$. Mais ce peut être autre chose, et notamment la subjectivité d'un personnage qui vient donner une assise - celle que donne le point de vue - à l'Imp qui en manque. C'est singulièrement le cas lorsque l'imparfait est, avec le PS qui le précède, en relation non d'inclusion mais de progression, avec un verbe atélique (de type état ou activité), comme dans (12) ou dans (22):

(22) [la fille], en quelques secondes, tira du coffre un vieux rideau qu'elle assujettit devant la petite vitre rectangulaire. Le jour traversait un peu le tissu bleuâtre et donnait un aspect de caverne à la soupente.

« Le lit va grincer, dit Jacquemort décidant de remettre sa psychanalyse à un peu plus tard » (Vian, L'Arrache-cour).

Dans cette occurrence on a, d'un point de vue temporel, la progression : [assujettit < traversait, donnait]. Les deux imparfaits traversait et donnait manquent sensiblement de point d'ancrage dans la mesure où nos connaissances du monde excluent la relation d'inclusion avec le PS qui les précède : ce n'est qu'après que la fille a disposé un vieux rideau que le jour traverse ledit rideau et transforme la soupente en caverne. On leur en donne un en interprétant les procès traversait et donnait comme vus par le (ou les) personnage(s) ${ }^{13}$.

A l'inverse, le PS, forme autonome, ne demande pas un adjuvant pour le fixer : il n'appelle donc pas «naturellement» un point de vue, qui, pour se manifester, devra être construit explicitement par le cotexte, ou répondre à une exigence de cohérence de celui-ci comme dans (19) et (21).

La clé de l'affinité de l'Imp avec le point de vue, comme du peu d'appétence du PS pour ce lien, tient donc, selon mon hypothèse, au fait que l'IMP, de par l'instruction aspectuelle [incidence] qu'il délivre, demande de construire le procès par rapport à un repère, ce repère pouvant être un point de vue ; alors que le PS, de par l'instruction aspectuelle [+ incidence] qu'il délivre, est son propre repère, et comme tel, ne lorgne pas du côté du point de vue.

\section{Conclusion}

Après avoir mis en perspective la métaphore scopique dans le traitement de l'aspect verbal et fait une analyse critique de l'usage de la notion de point de vue dans la littérature sur la question, je me suis attaché à montrer la relation entre l'instruction aspectuelle délivrée par le temps verbal en langue et la construction d'un point de vue qui peut lui être associé en discours. Il apparaît, au terme de cette étude, que

- la notion de point de vue, appliquée à la question du temps verbal, n'a qu'une pertinence métaphorique au niveau de la description des faits de langue, susceptible de s'avérer inadéquate dans le traitement de certaines occurrences : l'Imp ne saurait être défini

\footnotetext{
${ }^{13}$ Cette question du point d'ancrage subjectif de l'imparfait a fait l'objet de discussions pour le traitement de l'imparfait narratif. L. Tasmowski-De Ryck pose que dans :
}

(23) La clé tourna dans la porte d'entrée. M. Chabot retirait son pardessus qu'il accrochait au portemanteau, pénétrait dans la cuisine et s'installait dans son fauteuil d'osier (Simenon, La Danseuse du gai moulin).

c'est parce qu'on peut rétablir cotextuellement une perception - celle de la mère et du fils attendant le retour du père - que l'imparfait est possible $(1985: 75)$. Ce que confirme Vet: « without this context the PS would be obligatory in this fragment $\gg(1991: 23)$. Cette analyse - excessive selon moi (cf. des exemples comme (16), ainsi que Bres 2004a) - pointe sur une demande (qui peut ne pas être satisfaite) : celle de rattacher l'imparfait à un autre élément, par défaut à un point de vue. 
par la possibilité de construire une subjectivité scopique, pas plus que le PS par l'impossibilité d'une telle construction. Procéder ainsi reviendrait à court-circuiter la valeur en langue pour promouvoir un effet de sens textuel dans lequel le temps verbal est au mieux partie prenante mais qui ne lui appartient pas en propre ;

- la notion de point de vue a, en revanche, toute sa pertinence au niveau de la description de certains fonctionnements en discours des temps verbaux. Le point de vue (subjectif) est un des effets de sens à la production duquel le temps verbal participe en interaction avec différents éléments du co(n)texte. Il le fait à partir de sa valeur aspectuelle et en accord avec elle, ce qui explique que l'Imp soit plus facilement focalisable que le PS.

\section{Bibliographie}

Berthonneau A.-M. et Kleiber G., 1993. «Pour une nouvelle approche de l'imparfait : l'imparfait, un temps anaphorique méronomique », Langages 112, 55-73.

Berthonneau A.-M. et Kleiber G., 1999, « Pour une réanalyse de l'imparfait de rupture dans le cadre de l'hypothèse anaphorique méronomique », Cahiers de praxématique 32, 119-166.

Bres J., 1999, Compte rendu de Moeschler J. et al. (éd.), 1998, Le temps des événements, Cahiers de praxématique 33, 226-230.

Bres J., 2003, «Mais oui, il était un joli temps du passé comme les autres, mon joli petit hypocoristique... », Langue française 138, 111-125.

Bres J., 2004a, L'imparfait dit narratif (à paraitre).

Bres J., 2004b, «Sémantique de l'imparfait : dépasser l'aporie de la poule aspectuelle et de l'œuf anaphorique ? Eléments pour avancer », Cahiers Chronos (à paraître).

Brunot F. et Bruneau C., 1947/1969, Précis de grammaire historique de la langue française, Paris : Massin et Cie.

Combettes B., 1992, L'organisation du texte, Centre d'analyse syntaxique de l'université de Metz.

Comrie B., 1976, Aspect, Cambridge : Cambridge University Press.

Ducrot, O. (1984), «Esquisse d'une théorie polyphonique de l'énonciation », in Le dire et le dit, Paris : Minuit, pp. 171-233.

Fleischman S., 1991, "Verb tense and point of view", in Fleischman S et L. Waugh (éd.), Discourse pragmatics and the verb, London: Routledge, 26-54.

Genette G., 1972, Figures III, Paris : Editions du Seuil.

Gosselin L., 1996, Sémantique de la temporalité en français, Louvain-la Neuve : Duculot. Guillaume G, 1929/1970, Temps et verbe, Paris : Champion.

Haillet P., 2002, Le conditionnel en français : une approche polyphonique, Paris : Ophrys.

HoltJ., 1943, Etudes d'aspect. Acta Jutlandica 15.2.

Kamp H. et C. Rohrer, 1983, « Tense in texts », in R. Bauerle, C. Scharze et A. von Stechow (éds) : Meaning, use and interpretation of language, Berlin : De Gruyter, 250-269.

Kleiber G., 1993, «Lorsque l'anaphore se lie aux temps grammaticaux », in C.Vetters (éd.) : Le temps, de la phrase au texte, Presses universitaires de Lille, 117-166.

Kleiber G., 2003, "Entre les deux mon cœur balance, ou l'imparfait entre aspect et anaphore », Langue française 138, 8-19.

Labelle F., 2002, «Point de vue et aspect en français et en anglais », Cahiers Chronos 9, 7189.

Langacker R. W., 1987, Foundations of cognitive grammar, vol. 1, Stanford: Stanford University Press.

Lakoff G. et Johnson M., 1980/1985, Les métaphores dans la vie quotidienne, Paris : Editions de Minuit. 
Leeman D., 2003, «Le passé simple et son cotexte : examen de quelques distributions », Langue française 138, 20-34.

Martin R., 1971, Temps et aspect. Essai sur l'emploi des temps narratifs en moyen français, Paris : Klincksieck.

Molendijk A., 1990. Le passé simple et l'imparfait: une approche reichenbachienne, Amsterdam : Rodopi.

Nølke H., 1993, Le regard du locuteur. Pour une linguistique des traces énonciatives. Paris : Kimé.

Nølke H. et Olsen M., 2003, «Le passé simple subjectivisé », Langue française 138, 75-85.

Rabatel A., 1998, La construction textuelle du point de vue, Lausanne: Delachaux et Niestlé.

Rabatel A., 2003, «Une lecture énonciative des valeurs aspectuo-temporelles et commentatives de l'imparfait dans les suites PS + Imp : point de vue du locuteur ou de l'énonciateur », Journal of French Language Studies, 13-3, 1-17.

Saussure (de) L. et Sthioul B., 1999, «L'imparfait narratif: point de vue (et images du monde) », Cahiers de praxématique 32, 167-188.

Smith C. S., 1991, The parameter of aspect, London : Kluwer Academic Publishers.

Sthioul B., 1998 «Temps verbaux et points de vue », in Moeschler J. et al. (éd.), Le temps des événements, Paris : Kimé, 197-220.

Sthioul B., 2000, «Passé simple, imparfait et sujet de conscience », Cahiers Chronos 6, 7993.

Tasmowski-De Ryck L., 1985. «L'imparfait avec et sans rupture », Langue française 67, 5977.

Todorov T., 1968, «Les catégories du récit littéraire », Communications 8, 125-151.

Vet C., 1991, "The temporal structure of discourse : setting, change and perspective », in Fleischman S et L. Waugh (éd.), Discourse pragmatics and the verb, London: Routledge, 725.

Victorri B. et Fuchs C., 1996, La polysémie. Construction dynamique du sens, Paris : Hermès. Vogeleer S., 1994, «Le point de vue et les valeurs des temps verbaux », Travaux de linguistique 29, 39-58.

Weinrich H., 1964/1973. Le temps. Le récit et le commentaire, Paris : Le Seuil. Yvon H., 1926, L'imparfait de l'indicatif en français, Paris : Les Belles Lettres. 